\title{
De la mystification à la fiction. La poétique suicidaire de la fausse traduction
}

\author{
par \\ David MARTENS \\ Katholieke Universiteit Leuven
}

Par son retentissement, la plus notoire des fausses traductions de l'histoire littéraire moderne en Occident reste sans conteste le corpus ossianique publié par James MacPherson à partir de 1760. Mais, des Lettres d'une religieuse portugaise attribuées à Guilleragues au Romain Gary des Têtes de Stéphanie (1974) en passant par le Mérimée du Théâtre de Clara Gazul (1825) et de la Guzla (1827), le Pierre Louÿs des Chansons de Bilitis (1894) et les romans noirs pour l'un, coquins pour l'autre, publiés par Boris Vian et Raymond Queneau sous les noms, respectivement, de Vernon Sullivan (1946) et de Sally Mara (1947), la modernité littéraire française est loin de faire pâle figure au sein de cette tradition. Elle ne manque en effet pas de ces œuvres au statut ambigu, fondées sur une scénographie conjuguant la pseudo-traduction avec une stratégie mystificatrice dont le dévoilement confère à ces textes un statut fictif particulier.

En raison de la pratique dont ils ressortissent, de tels livres occupent au sein de l'institution littéraire une position marginale surdéterminée. Déviante au regard d'une norme dont elle mime certains attendus - à s'y méprendre parfois -, la mystification semble minoritaire par principe; elle n'a d'ailleurs jamais été reconnue comme genre à part entière, et pour cause, dans la mesure où elle consiste, 
précisément, à imiter les protocoles de présentation de genres constitués ${ }^{1}$. Ce caractère mineur se révèle plus prononcé encore lorsque ces textes se font passer pour d'authentiques traductions, c'est-à-dire se donnent à appréhender comme relevant d'une pratique traditionnellement tenue pour ancillaire par rapport à l'être-œuvre de l'œuvre et à la qualité d'original qui lui est associée. Enfin, quantitativement rares dans l'histoire littéraire, ces textes sont a fortiori minoritaires au sein du corpus de textes constitué par les traductions.

Encore peu étudiées, les pseudo-traductions ne concernent cependant pas un domaine sectoriel et secondaire de la traductologie. $\mathrm{Si}$ cette dernière s'est principalement développée à travers l'étude de «véritables» traductions, les interrogations que ne laissent pas de susciter les fausses traductions touchent au cœur de certains enjeux déterminants de la pratique d'écriture à laquelle elles feignent d'appartenir. En raison du simulacre en fonction duquel elles se donnent (se sont données) à lire, elles mettent en question les éléments de scénographie sur lesquels se fonde le statut de ce type d'écrits, caractérisés par l'instauration d'un rapport particulier entre un original et le texte considéré, présenté comme sa traduction dans une autre langue. Les pseudotraductions invitent à ce titre à une interrogation sur cette configuration textuelle spécifique que constitue la traduction, à travers les incidences de son inscription dans une poétique de la mystification.

Il s'agira de cartographier certaines des lignes de force de ces stratégies mystificatrices qui tendent à fictionnaliser un dispositif paratextuel destiné à faire passer un original pour une traduction. Alors que les pseudo-traductions «deviennent» des originaux à travers le dévoilement du simulacre en fonction duquel elles se donnent à lire, le dispositif mystificateur de la traduction acquiert une dimension fictive par la révélation de sa nature. Pour autant, le statut de traduction de ces textes ne se trouve pas simplement neutralisé par sa fictionnalisation. Ainsi, il rend problématique leur nouveau statut d'originaux en les maintenant sous l'emprise du paradigme de la traduction. Les deux

1 Sur la mystification littéraire, voir JEANDILlou (J.-Fr.), Esthétique de la mystification. Tactique et stratégie littéraire, Les Éditions de Minuit, Paris, coll. «Propositions », 1994, ainsi que son anthologie Supercheries littéraire. La Vie et l'œuvre des auteurs supposés (1989), Droz, Genève, 2001. 
moments auxquels sont soumis de tels dispositifs témoignent de cette ambiguïté statutaire, qui rend particulièrement problématique la traduction de ces pseudo-traductions. Leur caractère factice fausse en effet par contagion les traductions dont elles pourraient faire l'objet mettant en question les attendus de la conception courante du traduire.

\section{De la mystification à la fiction}

En 1825, peu avant l'avènement du romantisme en France, le jeune Prosper Mérimée publie Le Théâtre de Clara Gazul, recueil de pièces de théâtre prétendument traduites de l'espagnol, avant de récidiver en 1827 avec La Guzla ou Choix de poésies illyriques recueillies dans la Dalmatie, la Croatie et l'Herzégowine. En 1946, Boris Vian commet quant à lui J'irai cracher sur vos tombes, premier des quatre romans qu'il publiera sous le nom de Vernon Sullivan, un supposé romancier noir américain. L'année suivante, Raymond Queneau, publie On est toujours trop bon avec les femmes, roman attribué à une certaine Sally Mara et présenté comme une traduction de l'irlandais. Si en ce qui concerne le best-seller au parfum de scandale publié par Vian ce sera la critique qui dévoilera le pot-aux-roses, Mérimée et Queneau endosseront pour leur part de leur propre initiative la paternité de ces mystifications, le premier en 1842, le second en 1962.

Encore que selon une crédibilité relative, de tels textes se sont dans un premier temps fait passer pour ce qu'ils n'étaient pas, à savoir des traductions. Ce faisant, ils se sont présentés à leurs lecteurs sous le signe d'une double altérité, construite de toute pièce. Conçue comme la transposition d'un texte d'une langue dans une autre, la traduction se fonde sur un agencement (d'au moins) deux textes, ou de deux versions d'un «même» texte. Elle se donne par définition à lire comme hantée par le fantôme d'un autre, «l'original», avec lequel elle entretient un rapport fréquemment tenu pour ancillaire. Corollairement, la traduction se signale par l'établissement d'un rapport, lui aussi hiérarchisé, entre deux signatures, celle du traducteur étant le plus souvent subordonnée à celle de l'auteur, topographiquement et typographiquement, selon une procédure qui manifeste la minoration coutumière de la tâche du traducteur dans la prise en considération de l'économie de ce type de texte. 
Mérimée, Vian ou Queneau ont déployé des trésors d'ingéniosité en vue de garantir l'effet d'authenticité de ces textes fondés sur une imitation des protocoles de présentation de la traduction, c'est-à-dire de certains de ses éléments paratextuels tenus pour caractéristiques. Qu'il s'agisse des «Notice» ou «Préface» destinées à conférer une épaisseur biographique à l'auteur et au traducteur imaginaires, de notes lexicographiques, littéraires et historiques, de la publication dans des collections publiant de véritables traductions, ou encore de l'imitation de la présentation formelle de ce type de textes, ces signes dévolus à la crédibilisation de la supercherie ont contribué, avant de se voir reconfigurés par l'apposition d'embrayeurs de fictionnalité, à faire passer ces œuvres pour des traductions authentiques. Un Boris Vian ira même, afin de donner le change quant à son rôle de traducteur, jusqu'à écrire et publier un faux original américain de son J'irai cracher sur vos tombes, incité il est vrai par un procès pour outrage aux bonnes mœurs ${ }^{2}$.

De tels livres sont toutefois susceptibles d'exhiber ou de voir exhibée leur nature. Loin d'être un accident qui leur surviendrait de façon contingente, cette éventualité se trouve inscrite en eux dès leur genèse, au moins à titre de possibilité. Ainsi, la supercherie du Théâtre de Clara Gazul fut-elle rapidement éventée, Mérimée ayant lu certaines pièces en public avant même la parution du recueil, court-circuitant ainsi les effets mystificateurs du procédé pour une part de ses lecteurs. De même, les aveux de paternité de Vian à propos de J'irai cracher sur vos tombes n'ont dévoilé qu'un secret de polichinelle, qu'une frange de la critique avait éventé dès la parution du roman. Quant à la publication des Euvres complètes de Sally Mara sous le nom de Queneau en 1962, elle n'a fait que confirmer ce que certaines notes ironiques d'On est toujours trop bon avec les femmes suggéraient en tournant en dérision le travail du supposé traducteur. La succession incongrue de trois «N. d. T. » qui clôt sur une incongruité en forme de clin d'œil le chapitre XVIII du roman en témoigne exemplairement:

2 Toutefois, compte tenu des circonstances particulières qui ont entouré la publication du livre, il semble relativement malaisé de faire la part de l'ironie qui sous-tend ce geste; en effet, Vian a été dès la parution du roman soupçonné - et dans certains cas accusé - d'en être l'auteur (voir ARnaud (N.), Dossier de l'affaire J'irai cracher sur vos tombes (1974), Christian Bourgois, Paris, 2006). 
L'esprit irlandais, on le sait, n'obéit pas aux règles du raisonnement cartésien, non plus qu'à celles de la méthode expérimentale. Ni français, ni anglais, mais assez voisin du breton, il procède par « intuition ». Caffrey ne pouvant ouvrir la lourde, eut donc l'ankou ${ }^{1}$ que quelqu'un se trouvait là [...]. Cette anschauung ${ }^{2}$ lui boucla immédiatement les tripes. Essuyant la sueur qui lui dégoulinait encore de la tronche [...] et, découvrant son devoir d'un seul coup d'un seul ${ }^{3}$, il résolut de rendre compte [...] de la découverte qu'il venait de faire.

Suivent ces trois notes de bas de page suivantes:

1. Celticisme pour « intuition». (N. d. T.)

2. Germanisme pour «ankou». (N. d. T.)

3. Gallicisme pour « anschauung ». (N. d. T. $)^{3}$

Toute mystification vit dans la hantise d'une révélation dont elle livre parfois d'emblée des amorces, à l'instar de Queneau dans cette suite de notes significativement ponctuée par un «en français dans le texte » à travers lequel ce roman signe en sous-main son appartenance à la langue de Voltaire. Ce type de textes témoigne d'une fascination prononcée pour les jeux de dissimulation, qui va de pair avec une jubilation du dévoilement. Tout se passe comme si une mystification n'était parfaitement accomplie que dévoilée comme telle - c'est-à-dire neutralisée dans ses effets de tromperie. Plus précisément, le double discours de la mystification est finalement toujours tranché lors de la révélation à laquelle elle se trouve vouée par principe. Et le dévoilement de la supercherie instaure un clivage temporel et fonctionnel en vertu duquel ces textes ne sont plus lus après la révélation comme ils l'étaient avant celle-ci. Un passage s'opère d'une scénographie à une autre, qui ne laisse pas d'affecter en profondeur le statut du texte.

Le dévoilement de la mystification comme telle fait glisser le dispositif dans la sphère de la «feintise ludique partagée ${ }^{4}$ », contraignant en toute rigueur le lecteur à y voir une fiction. Une fois connue leur nature de supercherie, de tels textes paraissent ne plus poser pro-

3 Queneau (R.), Les Euvres complètes de Sally Mara (1962), Gallimard, Paris, coll. «L'Imaginaire », 2003, p. 222.

4 Voir Schaeffer (J.-M.), Pourquoi la fiction?, Seuil, Paris, coll. «Poétique», 1999. 
blème: perdant leur statut de traduction, ils semblent rendus à leur identité d'originaux, et leur présentation comme traduction (le plus souvent, le protocole factice visant à les faire passer pour des traductions est conservé dans leurs rééditions), se voit reléguée au rang de fiction. S'opère en conséquence un transfert de genre: de la traduction à la mystification, pratiques apparentées en ce qu'elles ne relèvent pas des genres à part entière (une traduction de roman reste un roman comme une mystification de poèmes donne à lire des poèmes). Mais la logique spécifique d'une telle mutation statutaire complexifie la structure de ce type de textes. Située dans la sphère de la fiction, la fausse traduction acquiert une valeur d'original, problématique en ce que le texte demeure à plusieurs égards soumis au modèle formel de la traduction.

\section{Une origine toujours déjà différée}

Le principe de dévoilement impliqué par tout dispositif mystificateur détermine une transformation de l'identité des textes en jeu, c'està-dire de l'horizon d'attente en fonction duquel ils seront lus, tout particulièrement, en l'occurrence, de leur statut de traduction, qui passe de l'authenticité au fictif. Cette métamorphose n'implique cependant pas que, une fois désignés pour ce qu'elles sont, ces supercheries puissent être lues comme n'importe quelle fiction. Le dévoilement auquel elles sont soumises ne saurait faire en sorte que la mystification n'ait pas eu lieu, aussi déconstruite ait-elle pu être dès le départ dans ses effets proprement mystificateurs, par l'auteur luimême (Mérimée/Clara Gazul; Queneau/Sally Mara) ou par la critique (Vian/Vernon Sullivan). Procédant de la désignation d'un simulacre, ces textes préservent nécessairement en leur sein la mémoire de leur premier statut et cette dernière ne saurait être sans incidence sur la réception dont ils font désormais l'objet.

Les fausses traductions sont dans un premier temps sous-tendues par une «scène englobante» (rapport entre au moins deux figures d'auteur, deux langues, deux cultures à une époque déterminée), une «scène générique» (l'appartenance à un genre de textes particulier, 
mais aussi et surtout à cette pratique que constitue la traduction) et enfin une «scénographie» spécifique (le dispositif mystificateur singulier que met en œuvre un auteur de pseudo-traduction). Lorsque leur véritable nature est rendue publique, une scénographie seconde - le dévoilement, qui s'opère lui aussi à travers une scénographie particulière - partage avec le lectorat le caractère de feintise de la stratégie scripturaire adoptée ${ }^{5}$, de sorte que l'ensemble de l'élaboration textuelle s'en trouve reconfigurée: la «scène englobante» ne se voit pas réduite à rien mais se modalise dans les termes d'une fiction, de même que la «scène générique» par laquelle le texte s'est fait passer pour une traduction.

Lors de la publication d'une fausse traduction, le texte et sa signature se donnent certes dans un premier temps à lire comme autres que ce qu'ils sont effectivement. Mais ce "premier temps » procède d'un après-coup, en fonction duquel cette altérité postulée s'affiche comme leurre. A l'instar du secret tel que Louis Marin en décrit la logique, la mystification «ne se constitue tel[le] que de sa disparition; [elle] ne s'écrit qu'au futur antérieur ou, au mieux, à l'imparfait ${ }^{6} »$. La mystification «est dans l'évidence de son éclat aveuglant quand [elle] aura été $[\ldots]$ : c'est au moment où il n'y a plus de [mystification] que [la mystification] - si l'on peut dire - bat son plein ${ }^{7} »$. En l'occurrence, le devenir-autre du texte, le devenir-fiction de la traduction se conjugue sur le mode de l'avoir-été facticement, de l'être-passé-pour, qui affecte la mystification comme telle aussi bien que son objet. Les pseudo-traductions n'acquièrent en conséquence la valeur d' «œuvre» et d' «originaux» que dans le second temps que constitue le dévoilement de leur inauthenticité en tant que traductions.

Les fausses traductions ne naissent pas fictions, mais le deviennent par le geste à travers lequel se dévoile leur statut véritable, que celuici soit le fait de l'auteur ou de la critique. En conséquence, les fausses

5 Sur ces notions, voir Maingueneau (D.), Le Discours littéraire. Paratopie et scène d'énonciation, Armand Collin, Paris, coll. «U - Lettres», 2004, p. 190 et $s q$. Je remercie Ute Heidman de m'avoir suggéré l'intérêt de cet ouvrage pour rendre compte de la poétique la fausse traduction.

6 MARIN (L.), «Logiques du secret», in Lectures traversières, Albin Michel, Paris, coll. «Bibliothèque du Collège international de philosophie », 1992, p. 247.

7 Ibidem, p. 253. 
traductions diffèrent toujours déjà d'avec elles-mêmes. Leur apparent retour à leur identité propre s'avère par principe impossible, dans la mesure où elles n'ont jamais été et ne seront jamais quittes - c'est-àdire appréhendées indépendamment - de la supercherie qui a marqué leur publication originale. Paradoxalement, ce statut tenu pour véritable à la suite du dévoilement de leur nature n'est pas premier, puisque le texte n'a pas d'abord paru sous cette forme. Il ne saurait dès lors reconquérir par là une quelconque forme d'originalité. Il ne saurait dès lors conduire le texte à reconquérir une quelconque forme d'originalité. Les valeurs d'authenticité et d'originalité dont ce type d'écrits est affecté changent d'objet: le devenir-mystification de ces (fausses) traductions, solidaire d'un devenir-original, ne va pas sans reste: il ne s'agit certes plus d'authentiques traductions, mais, tout de même, de véritables fausses traductions.

La relation dynamique entre deux textes - dont l'un, l'original, se révèle imaginaire après-coup - qui caractérise les pseudo-traductions est régie par un agencement aux éléments mobiles, marqué par les modifications qu'il subit en fonction des deux temps et des deux scénographies agencées qui le déterminent. Ainsi, en dépit de la stratégie de réduction du texte à une supposée authenticité, une altérité scripturaire demeure. Bien qu'après le dévoilement de leur nature, de tels textes ne puissent plus, en droit, être lus comme ils l'étaient dans un premier temps, le principe de la traduction continue de les hanter, et non seulement sur le mode neutralisé d'une feintise ludique désormais partagée par un lecteur censé ne plus ignorer que le dispositif traducteur constitue une mystification. Certes, la démystification modifie le mode de lecture de la fausse traduction en donnant à lire un texte «original», mais il institue simultanément, au sein du «même» texte, la coexistence de deux scènes d'énonciation combinées et (successives), soit de deux versions concomitantes.

Leur nature véritable une fois dévoilée, ces pseudo-traductions préservent en effet, non pas malgré mais, bien au contraire, en raison de cette révélation même, la trace de leur premier mode de parution. Elles ne s'instituent comme mystifications que de la négation de celuici, c'est-à-dire de leur statut de traduction. Cette mémoire confronte le lecteur à deux versions différentes du «même» texte, selon une combinatoire formellement analogue - dans le schème qu'elle institue - à celle qui détermine les rapports entre une véritable traduction et son original. Pour le dire autrement, si l'original est rétabli - ou plutôt 
établi - en tant que tel par la révélation de la mystification dont il a été l'objet, deux versions du «même» texte coexistent en effet, dans la même langue : celle où la feintise est partagée, celle où elle ne l'est pas (ou plutôt ne l'a pas été), la seconde étant impliquée par la précédente comme l'original dans sa traduction (même si c'est cette dernière qui est marquée au coin de l'inauthenticité).

A première vue identiques en tous points, ces deux versions diffèrent dans le même temps de façon radicale. Certes, hormis l'élément paratextuel qui assure le dévoilement de la supercherie, rien ne distingue leur lettre. Mais le changement d'identité de l'auteur et éventuellement du traducteur, ainsi que celui du statut du texte en bouleversent l'horizon d'attente et d'interprétation: selon que Le Théâtre de Clara Gazul et La Guzla soient signés par Mérimée ou, respectivement, par une jeune actrice espagnole ou un joueur de guzla originaire des Balkans, selon que J'irai cracher sur vos tombes soit signé par Boris Vian ou un noir américain, selon que les livres de Sally Mara soient signés par elle-même ou par Raymond Queneau, il ne s'agit plus, en toute rigueur, des mêmes textes. Non seulement parce que les coordonnées de la fonction-auteur divergent, mais aussi parce que lorsqu'ils reprennent leurs livres sous leur signature et dans leur langue, Mérimée, Vian et Queneau signent non seulement ces textes, mais aussi la mystification dont ils ont fait l'objet.

La structure de relevance qui détermine les deux temps de la supercherie n'abolit pas le bibelot pour lequel peut passer la traduction lorsqu'elle contribue à scénographier une mystification. Paraissant récuser leur appartenance à l'ordre de la traduction à la faveur du dévoilement de leur statut, ces supercheries la préservent non seulement sous la forme neutralisée de la feintise ludique partagée, mais elles en transfèrent également la logique sur le plan du dispositif mystificateur, de telle façon que son dévoilement le reconfigure en le donnant à lire pour ce qu'il est effectivement. Deux versions du «même» texte coexistent par principe dans la mystification, selon un schème qui la désigne comme un lieu d'expérimentation de ses limites particulièrement propice pour la traduction. En effet, la préservation de cette bipolarité constitutive pose problème lorsqu'il s'agit d'envisager la traduction de tels textes. Ironisant sur le travail de la traduction, ils en remettent en question les fondements, tout spécialement lorsqu'il s'agit de les traduire dans la langue de leur original imaginaire. 


\section{Comment traduire une fausse traduction?}

Dans le texte traduit en français sous le titre «La tâche du traducteur», Walter Benjamin considère que si les œuvres originales sont susceptibles de connaître de multiples traductions, «[1]es traductions, en revanche, se révèlent intraduisibles ${ }^{8}{ }$. Certes, mais de nombreux exemples de traductions effectivement traduites au cours de la longue histoire de la traduction paraissent témoigner du contraire. Commentant le texte de Benjamin, Jacques Derrida précise que, si un original «peut se laisser de nouveau traduire et retraduire», en revanche, «[u]ne traduction, elle, ne le peut pas en tant que telle ${ }^{9} »$. En d'autres termes, les traductions paraissent intraduisibles en droit, mais peuvent parfaitement être traduites dans les faits. Selon une telle axiomatique, une traduction ne serait traduisible qu'en tant qu'autre et, plus précisément, en tant qu'original, soit qu'elle serve de relais pour une traduction de l'original dans une autre langue, soit que, à l'instar de celles de Sophocle par Hölderlin, elle ait acquis un statut d'original à part entière.

Mais qu'en est-il des fausses traductions, dont l'identité, à cet égard, est pour le moins équivoque? Qu'advient-il de ces textes lorsqu'ils se trouvent amenés à subir l'épreuve de cette étrangeté, inquiétante (en même temps qu'amusante à l'occasion), que constitue la traduction? Comme nous l'avons vu, une fois révélée leur qualité d'originaux, ils demeurent des traductions, ne fût-ce que parce qu'ils en préservent formellement les traits caractéristiques. Or, comment traduire un texte qui est à la fois un original et une traduction, un original qui, formellement, se présente comme une traduction? La question semble de pure rhétorique, dès lors que le dévoilement de la supercherie fait de ces textes, en même temps que des fictions, des originaux à part entière. Pourtant, à l'usage, leur traduction ne laisse pas de poser problème. En témoigne la seconde préface de La Guzla,

8

Benjamin (W.), «La tâche du traducteur» (1923), in Euvres, t. I, trad. de l'allemand par M. de Gandillac, R. Rochlitz et P. Rusch, présentation par R. Rochlitz, Gallimard, Paris, coll. «Folio Essais », 2000, p. 260-261.

9 Derrida (J.), «Des tours de Babel» (1985), in Psyché. Inventions de l'autre (1987), Galilée, Paris, coll. «La philosophie en effet », nouvelle édition augmentée, 1998, 2 tomes, p. 225. 
qui accompagne la réédition de l'ouvrage en 1842, et dans laquelle Mérimée ironise savoureusement sur les traductions de son livre entreprises par certains de ses plus prestigieux lecteurs.

Deux mois après la publication de la Guzla, M. Bowring, auteur d'une anthologie slave, m'écrivit pour me demander les vers originaux que $\mathrm{j}$ ' avais si bien traduits.

Puis M. Gerhart, conseiller et docteur quelque part en Allemagne, m'envoya deux gros volumes de poésies slaves traduites en allemand, avec la Guzla traduite aussi, et en vers, ce qui lui avait été facile, disait-il dans sa préface, car sous ma prose il avait découvert le mètre des vers illyriques. Les Allemands découvrent bien des choses, on le sait, et celui-là me demandait encore des ballades pour faire un troisième volume.

Enfin, M. Pouchkine a traduit en russe quelques-unes de mes historiettes, et cela peut se comparer à Gil Blas traduit en espagnol, et aux Lettres d'une religieuse portugaise, traduites en portugais ${ }^{10}$.

Savamment graduée, l'ironie mériméenne se fonde sur le présupposé en vertu duquel une traduction serait intraduisible en tant que telle. Des traductions de traduction comme celles de Gerhart et Pouchkine viseraient, à travers le texte de La Guzla, le supposé original en langue illyrique et non la traduction pour elle-même (à en croire Mérimée, les deux premiers lecteurs évoqués lui auraient d'ailleurs demandé de leur procurer l'original). Or, en raison de la méprise en fonction de laquelle ces deux traducteurs ont appréhendé et traduit le texte de Mérimée, leurs traductions se sont trouvées d'emblée faussées par la méconnaissance du statut véritable du texte qu'elles prenaient en charge. La révélation du statut effectif de la supercherie mériméenne souligne en effet que, si ces traductions se présentent comme des traductions d'une traduction, elles fournissent aux lecteurs allemands et russes une information erronée sur elles-mêmes, en ce qu'elles sont en réalité, à l'insu de leurs auteurs, des traductions directes d'un original en français.

La mystification affecte le statut des traductions publiées par Pouchkine de façon plus prononcée encore que celles de son homo-

10 MÉRIMÉE (P.), La Guzla ou Choix de poésies illyriques recueillies dans la Dalmatie, la Bosnie et l'Herzégowine, Kimé, Paris, coll. « Rencontres », 1994, p. 25. 
logue allemand. Rapprochées de celle du Gil Blas de Santillane de Lesage en espagnol et de celle des Lettres d'une religieuse portugaise en portugais, elles font figure de comble au sein de la scénographie ironique agencée par Mérimée. A suivre la logique de cette dernière, cette traduction en russe consisterait en effet en une réécriture de l'original effectuée à l'insu du traducteur. Le travail aurait consisté mais il y aurait tout de même lieu de s'interroger sur les motifs qui conduisent Mérimée à identifier l'illyrique de La Guzla avec la langue russe, qu'il apprendra sur le tard -, à traduire ces textes dans la langue de leur version originale supposée. Par conséquent, le statut de traduction du texte de Pouchkine, qu'il justifie par ailleurs parfaitement, se voit dénié dans la mesure où il résulterait d'une (re)production de l'original (imaginaire) à partir de sa supposée traduction.

Les lecteurs et les traducteurs abusés par une pseudo-traduction peuvent certes faire l'objet de moqueries plus ou moins faciles. Mais ces textes piégés posent également des problèmes aux traducteurs qui connaîtraient leur nature véritable, surtout s'il s'agit de les traduire dans leur langue originale supposée par la scénographie mystificatrice. En effet, ces supercheries postulent non seulement un original, mais vont en certaines occasions jusqu'à le citer, notamment à travers des notes lexicographiques qui visent à expliciter le sens de certains termes. Or, dans la mesure où le dispositif de la traduction se trouve fictionnalisé et que la qualité d'original lui est assignée à la faveur du dévoilement de la nature véritable du texte, il s'agit de tout traduire, notes comprises. Traduire une fausse traduction implique par conséquent l'annotation de ce type de notes, afin de préserver dans la traduction la différence de langues inscrite dans l'original. Or, comme le note Derrida, «[q]uand un "original" parle de sa langue en parlant sa langue il prépare une sorte de suicide à la traduction, comme on dit suicide au gaz ou suicide par le feu ${ }^{11}{ }^{»}$.

De même, lorsqu'une fausse traduction cite son prétendu original, elle contraint son traducteur dans cette langue originale à un suicide $\grave{a}$ la traduction, voire de la traduction. Une telle traduction, donnant

11 Derrida (J.), «S'il y a lieu de traduire. I. La philosophie dans sa langue nationale (vers une "licterature en françois") », in Du droit à la philosophie, Galilée, Paris, coll. «La philosophie en effet», 1990, p. 309. 
corps à l'original imaginaire postulé par la pseudo-traduction, ne saurait, par définition, traduire ces citations de l'original fictif. Elle se verra donc ponctuellement contrainte à passer son chemin, à ne pas traduire, se bornant à recourir à la citation. Mais elle est dans le même temps tenue, si elle tient à rendre compte autant que possible de la construction particulière de l'original et de la différence des idiomes qui le caractérise - cela doit faire partie de son ambition en tant que traduction -, de notifier au lecteur ce suspend localisé du geste traducteur. Or, ce faisant, elle transgresserait la loi économique qui régit, selon Derrida, la conception traditionnelle de la traduction. En de pareilles circonstances, écrit le philosophe, «[i]l faut ou bien se résigner à [...] perdre l'effet, l'économie, la stratégie [de la traduction] (et cette perte peut être énorme) ou bien au moins y ajouter une glose, du type N.d.T., qui toujours, même dans le meilleur des cas [...], avoue l'impuissance ou l'échec de la traduction [et] rompt avec [...] la loi économique du mot, qui définit l'essence de la traduction au sens strict, de la traduction normale, normée [...]. Partout où l'unité du mot est menacée ou mise en question, ce n'est pas seulement l'opération de la traduction qui se trouve compromise, c'est le concept, la définition et l'axiomatique même, l'idée de la traduction qu'il faut reconsidérer ${ }^{12} »$.

Souhaitant traduire dans la langue de leur original imaginaire les fausses traductions de Mérimée, de Vian ou de Queneau en préservant cette «loi économique du mot, qui définit» selon Derrida «l'essence de la traduction au sens strict», un traducteur téméraire verrait deux options distinctes s'offrir à lui, à travers lesquelles sa traduction fraierait les voies d'un échec programmé par la structure pour le moins retorse, voire perverse, du texte à traduire.

Ce traducteur pourrait préserver le dispositif paratextuel propre à une traduction en annotant les notes qui accompagnent l'original et le citent dans sa langue pour crédibiliser la supercherie. Mais à travers une telle stratégie, il transgresserait la loi économique de la traduction en redoublant un jeu de notes marquant déjà - bien que sur le mode du

12 Derrida (J.), «Qu'est-ce qu'une traduction "relevante"?», in Jacques Derrida, sous la dir. de M.-L. Mallet et G. Michaud, L'Herne, «Cahiers de l'Herne » $\mathrm{n}^{\circ}$ 83, Paris, 2004, p. 565. 
simulacre - l'échec - ou du moins le caractère factice - de la traduction au sens strict. Il s'agirait en effet de redoubler certaines notes, ce qui n'arrive normalement pas dans les traductions de traduction, où ces éléments sont gommés dans la mesure où c'est l'original qui est visé. Dès lors, une traduction en castillan de certaines notes lexicographiques du Théâtre de Clara Gazul supposerait des notations du type «en castellano en el texto », appelées par les notes de l'édition originale du texte citant des termes issus de la langue de Cervantès.

Afin de tenter autant que faire se peut de préserver l'économie du mot à mot supposée caractériser la traduction, ce traducteur pourrait également inscrire la scénographie mystificatrice dans une tout autre relation de pays et un tout autre rapport de langues que ceux qui régissent la supercherie dans sa version originale. Le Théâtre de Clara Gazul pourrait ainsi se voir traduit en espagnol à partir d'un original présenté, par exemple, comme portugais. Toutefois, aussi intéressante puisse-t-elle se révéler, une telle tentative ne produirait pas une traduction à proprement parler. Elle relèverait davantage d'une réécriture en ce qu'elle perdrait non seulement la lettre de l'original, mais aussi la spécificité du rapport entre deux langues et deux cultures à une époque donnée qui singularise la supercherie originale. Sans compter qu'un tel traducteur (supposons-le honnête...) serait en outre tenu de signifier son opération sous la forme d'une note du type de celle que sa stratégie scripturaire visait précisément à éviter...

De ces deux formes de suicide de la traduction, plus que probablement, la solution la plus couramment pratiquée sera la première. Bien qu'elle multiplie les prothèses par lesquelles la traduction signifie les limites de l'idéal qui la porte, il s'agit de la stratégie qui en demeure la plus proche. Mais l'échec ou la contrainte suicidaire qui marque ces deux options paraissant borner un certain idéal de la traduction témoigne de ce que, bien que ces supercheries soient en toute rigueur des originaux, leur imitation des protocoles de la traduction rend impossible de faire droit au principe économique qui régit cette dernière. Toute tentative d'un traducteur pour s'y plier faussera de telles traductions de fausses traductions en les contraignant à inscrire en leur sein l'échec - ou le suicide - programmé par le caractère mystificateur du 
texte source. Marquées par le caractère de traductions des textes qu'elles traduisent, ces traductions de fausses traductions sont condamnées à être, elles aussi, du moins en droit, de fausses traductions ${ }^{13}$.

\section{Aux limites de la traduction}

Les fausses traductions suggèrent que rien ne permet, intrinsèquement, de distinguer de façon certaine un original d'une traduction, autrement dit qu'une traduction n'est jamais instituée comme telle que parce qu'elle s'autorise d'elle-même en configurant une scène d'énonciation d'un type particulier. Une traduction ne se désigne en effet comme telle et ne soutient ce performatif auto-instituant qu'en fonction de procédures qu'il est loisible à un écrivain habile de feindre de façon plus ou moins convaincante. Mais une fois dévoilée la supercherie, les pseudo-traductions demeurent marquées par leur premier mode de parution; leur transformation statutaire se fonde sur un mouvement de «relevance» qui préserve la trace de la négation qu'il opère et de ce qu'elle déniait. Même lorsqu'elles paraissent récuser leur appartenance effective à l'ordre de la traduction, les pseudotraductions en maintiennent le schème sur le plan du dispositif, comme en témoignent les problèmes posés par leur traduction, en particulier dans leur langue d'origine supposée.

Pourtant, bien qu'impossibles en droit, de telles traductions sont effectivement réalisables. L' on a bel et bien pu traduire ces textes dans leur langue d'origine fictive, comme Le Théâtre de Clara Gazul en castillan. Il n'en reste pas moins que la stratégie mystificatrice, lorsqu'elle recourt à une scénographie de traduction, marque sa traduction de son sceau falsifiant, puisqu'elle la conduit à se dénaturer en la confrontant à l'impossibilité de respecter de façon stricte la loi économique en vertu de laquelle elle se trouve le plus souvent envisagée. Les pseudo-traductions contraignent leurs traducteurs à la glose, c'està-dire à l'interruption du geste censé les qualifier en propre. Ce genre

13 Pour un prolongement des présentes réflexions à travers une étude de cas concrets, voir MARTENS (D.), Au miroir de la pseudo-traduction. Ironisation du traduire et traduction de l'ironie, dans Linguistica antverpiensia, new series, $\mathrm{n}^{\circ} 9$, «Traduire l'ironie», s. dir. Lievois (K.) et Schoentjes (P.), à paraître en 2010. 
qui n'en est pas un, et qui n'appartient pas sans problème à cette forme d'écriture particulière que constitue la traduction, fausse toute traduction: la fausse traduction voit son caractère factice affecter non seulement, en amont, les textes dont elle modalise la lecture, mais aussi, en aval, les tentatives de traduction dont elle est susceptible de faire l'objet.

Les fausses traductions paraissent ainsi doublement étrangères à la traduction en tant que telle. Outre qu'il ne s'agit pas d'authentiques traductions (elles ne procèdent pas d'un original), elles menacent toute tentative de traduction de ratage. Dans le même temps, elles réalisent l'idéal suicidaire de la traduction, soit l'identification parfaite et (presque) sans reste de la traduction à son original (le reste en question résidant, en l'occurrence, dans le geste de dévoilement relatif au statut mystificateur du texte). Selon un tel point de vue, ces textes apparaissent à certains égards comme étant à eux-mêmes leur propre traduction, c'est-à-dire la réduction à rien de leur être-traduction; le passage qu'ils subissent du statut de traduction à celui d'original ne modifie en rien leur lettre: en tant qu'originaux, ils coïncident parfaitement avec la traduction pour laquelle ils se sont fait passer. Mais, ne mettant plus deux idiomes en relation, ils échappent dès lors à la sphère de la traduction au sens restreint (d'une langue à une autre).

Reste que cette poétique suicidaire de la pseudo-traduction se trouve elle-même condamnée au ratage, puisque ces textes participent néanmoins du paradigme de la traduction. Tout se passe comme s'ils performaient et permettaient ainsi de toucher du doigt l'impossibilité dans laquelle ils se trouvent de rompre définitivement avec leur appartenance (aussi problématique soit-elle) à l'ordre de la traduction, alors même que leur lettre ne procède pas d'un geste de traduction. Ils invitent ainsi à repenser l'axiomatique la plus partagée à propos de la traduction, non à travers une réflexion théorique quant à son statut, mais bien à travers une performance et une scénographie particulière, qui conduisent à conférer à un dispositif censé donner à lire un texte comme une traduction le statut de fiction mystificatrice. Leur traitement au sein de ces fictions particulières que constituent les mystifications en révèle en effet sur l'être-traduction de la traduction et sur son 
rapport à l'original, en soulignant le caractère éminemment relatif d'une telle architectonique conceptuelle ${ }^{14}$.

Les pseudo-traductions permettent non seulement d'importer des pratiques scripturaires nouvelles au sein d'un champ littéraire ${ }^{15}$, mais elles conduisent aussi à reconsidérer les clivages hiérarchiques - notamment celui de l'ancillarité - en fonction desquels les traductions sont habituellement envisagées. Elles font surgir les limites de la traduction en tant que telle, de l'être-traduction de la traduction. Elles en participent de façon distanciée, puisqu'elles se caractérisent par le déni de leur statut de traduction, mais d'une façon pour le moins ambiguë, puisqu'elles en préservent la forme. A ce titre, la poétique particulière de ce type de textes brouille la frontière entre traduction et écriture à proprement parler, montrant, notamment, que toute traduction constitue, d'un certain point de vue, un original à part entière. Marqués au coin de fiction, ces textes donnent ainsi à appréhender en actes certaines des apories et des limites de la pratique scripturaire à laquelle ils feignent d'appartenir et à laquelle, de ce point de vue, ils appartiennent effectivement, en tant que cas-limites.

14 Étudiant également la pseudo-traduction, Emily Apter propose à cet égard un intéressant rapprochement entre la logique de la traduction et celle du clonage (voir «Translation with no originals: scandals of textual reproduction », in The Translation Zone : a New Comparative Literature, Princeton University Press, Princeton, 2006, p. 210-225).

15 Voir TOURY (G.), «Translation, literary translation and pseudotranslation », in Comparative Criticism, ${ }^{\circ}$ 6, 1984, p. 73-85 et «Enhancing cultural changes by means of fictitious translations », in Translation and Culturel Change, sous la dir. de A. Hung, Benjamins, Amsterdam and Philapdelphia, 2005, p. 3-18 (article consultable en ligne - URL: http://www.tau.ac.il/ toury/works/fict.htm), ainsi que LOMBEZ (Chr.), «La "traduction supposée" ou : de la place des pseudo-traductions poétiques en France», in Fictionalising Translation and Multilingualism, sous la dir. de D. Delabastita et R. Grutman, in Linguistica antverpiensa, $\mathrm{n}^{\circ} 4,2005$, p. $107-121$. 


\section{BIBLIOGRAPHIE}

APTER (E.), «Translation with no originals : scandals of textual reproduction », in The Translation Zone: a New Comparative Literature, Princeton University Press, Princeton, 2006, p. 210-225.

ARnaud (N.), Dossier de l'affaire J' irai cracher sur vos tombes, Christian Bourgois, Paris, 2006 [1974].

Benjamin (W.), «La tâche du traducteur», in Eeuvres, t. I, trad. de l'allemand par M. de Gandillac, R. Rochlitz et P. Rusch, présentation par R. Rochlitz, Gallimard, Paris, coll. «Folio Essais», 2000 [1923], p. 244-262.

Chelebourg (Chr.), Prosper Mérimée, le sang et la chair. Une poétique du sujet, Minard, Paris-Caen, coll. «Archives des Lettres modernes », 2004.

CoHN (D.), Le propre de la fiction, trad. de l'anglais par Cl. HarySchaeffer, Seuil, Paris, coll. «Poétique», 2001.

Collombat (I.), «Pseudo-traduction: la mise en scène de l'altérité », in Le Langage et l'homme, vol. XXXVIII, $\mathrm{n}^{\circ}$ 1, juin 2003, p. 145-156.

DERRIDA (J.), «Des tours de Babel»(1985), in Psyché.Inventions de l'autre, nouvelle édition augmentée, 2 tomes, t. 1, coll. «La philosophie en effet», Galilée, Paris, 1998 [1987], p. 203-235.

DERRIDA (J.), «S'il y a lieu de traduire. I. La philosophie dans sa langue nationale (vers une "licterature en françois") », in Du droit à la philosophie, Galilée, Paris, coll. «La philosophie en effet», 1990, p. 283-309.

DERRIDA (J.), «Qu'est-ce qu'une traduction "relevante"?», in Jacques Derrida, sous la dir. de M.-L. Mallet et G. Michaud, L'Herne, «Cahiers de l'Herne », n ${ }^{83}$, Paris, 2004, p. 561-576.

JEANDILlou (J.-Fr.), Esthétique de la mysification. Tactique et stratégie littéraire, Minuit, Paris, coll. «Propositions », 1994.

JeANDILlou (J.-Fr.), Supercheries littéraires, La Vie et l'œuvre des auteurs supposés, Droz, Genève, 2001 [1989].

LOMBEZ (Chr.), «La "traduction supposée" ou: de la place des pseudotraductions poétiques en France», in Fictionalising Translation and Multilingualism, sous la dir. de D. Delabastita et R. Grutman, Linguistica antverpiensa, new series, $\mathrm{n}^{\circ} 4,2005$, p. 107-121. 
LouŸs (P.), Les Chansons de Bilitis. Pervigilium mortis, édition présentée, établie et annotée par J.-P. Goujon, Gallimard, Paris, coll. «Poésie», 1990.

Maingueneau (D.), Le Discours littéraire. Paratopie et scène d'énonciation, Armand Collin, Paris, coll. «U - Lettres », 2004.

MARIN (L.), «Logiques du secret», in Lectures traversières, Albin Michel, Paris, coll. «Bibliothèque du Collège international de philosophie », 1992, p. 247-257.

MARTENS (D.), «Au miroir de la pseudo-traduction. Ironisation du traduire et traduction de l'ironie», dans Linguistica antverpiensia, new series, $\mathrm{n}^{\circ}$ 9, «Traduire l'ironie », s. dir. Lievois (K.) et Schoentjes (P.), à paraître en 2010.

MÉRIMÉE (Pr.), Théâtre de Clara Gazul, Romans et nouvelles, édition établie et annotée par J. Mallion et P. Salomon, Gallimard, Paris, coll. «Bibliothèque de la Pléiade », 1978.

MÉriméE (Pr.), La Guzla ou Choix de poésies illyriques recueillies dans la Dalmatie, la Bosnie et l'Herzégowine, introduction par A. Fonyi, Kimé, Paris, coll. « Rencontres », 1994.

PAVEL (Th.), Univers de la fiction, Seuil, Paris, coll. «Poétique», 1988 [1986].

Ost (Fr.), Traduire. Défense et illustration du multilinguisme, Fayard, Paris, coll. «Ouvertures », 2009.

Queneau (R.), Les ceuvres complètes de Sally Mara, Gallimard, Paris, coll. «L'Imaginaire », 2003 [1962].

SchaefFer (J.-M.), Pourquoi la fiction?, Seuil, Paris, coll. «Poétique», 1999.

SCHOOlCRAfT (R.), Romain Gary. The Man who sold his Shadow, University of Pennsylvania Press, Philadelphia, coll. «Critical Authors \& Issues », 2001.

TOURY (G.), « Translation, literary translation and pseudotranslation », in Comparative Criticism, $\mathrm{n}^{\circ}$ 6, 1984, p. 73-85.

TOURY (G.), «Enhancing Cultural Changes by Means of fictitious Translations ", in Translation and Culturel Change, sous la dir. de E. Hung, Benjamins, Amsterdam and Philapdelphia, 2005, p. 3-18; article consultable en ligne - URL : http://www.tau.ac.il/ toury/works/fict.htm.

VIAN (B.), J'irai cracher sur vos tombes, Christian Bourgois, Paris, 1973 [1946].

ViAn (B.), Les morts ont tous la même peau, Christian Bourgois, Paris, 1973 [1947]. 
